Int. J. Speleol. 19 (1990): 9-27

\title{
Secondary sympatric occurrence of sibling species of subterranean shrimps in the Karst
}

\author{
M. Cobolli Sbordoni*, M. Mattoccia**, G. La Rosa*, \\ E. De Matthaeis* and V. Sbordoni**
}

\begin{abstract}
SUMMARY
Allozyme variation at 32 gene loci was studied in cave dwelling shrimps of the genus Troglocaris (fam. Atyidae). Populations were collected from several caves and wells belonging to different drainage systems in the Karst areas of NE Italy. Results suggest the existence of two sympatric, genetically distinct, biological species (provisionally indicated as species A and B in the T. anophthalmus complex) in the samples collected at the Duino Well, Sagrado and Comarie caves (Nei's $\bar{D}=0.209$ ). Samples from Gradisca and Aurisina are represented by only one (A) or the other (B) of the two species, respectively. Two remaining eastern populations from the Rosandra Valley also proved to be monospecific. However, they possess fixed alternative alleles markedly different from both species $A$ and $B$ suggesting that these two populations may belong to a third sibling species, $C$ (A vs $C, \bar{D}=0.346 ; B$ vs $C, \bar{D}=0.146$ ). These data are discussed in relation to allopatric speciation of cave dwelling shrimp species, flooding periods of subterranean waters and the role of cave organisms as markers of this process.
\end{abstract}

\section{INTRODUCTION}

Speciation processes of cave dwelling populations and their divergence from epigean ancestors are thought to be promoted at a large extent, by geographic isolation (Barr, 1968; Sbordoni, 1982; Barr and Holsinger, 1985), although in some instances active dispersal accompanied by adaptive shift has been hypothesized as a possible speciation mechanism (Howarth, 1987; Rouch and Danielopol, 1987). Several studies carried out on the genetic structure of cave populations revealed an high rate of genetic differentiation between populations supposed to be conspecific on morphological ground (Sbordoni, 1982). In caves, according to Culver (1982), "many morphological species may contain several subspecies or even species". Italy

* Department of Animal and Human Biology, University of Rome "La Sapienza", ** Department of Biology, University of Rome "Tor Vergata", Italy. 


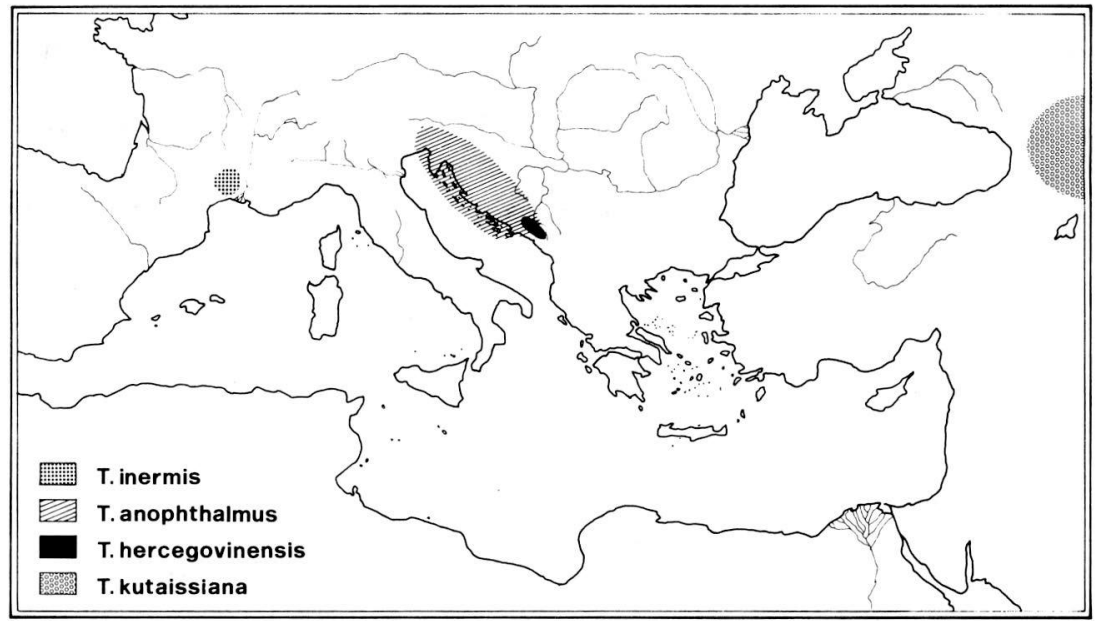

Fig. 1 - Range of the genus Troglocaris.

Reported examples of morphologically close or sibling species detected electrophoretically include cave millipeds (Laing et al., 1976), Speonomus beetles (Delay et al., 1980; Juberthie, 1988) and stygobiont Cyrolanid crustaceans (Caccone et al., 1986). In the above cases, due to the allopatry of such populations, their status as distinct species is only inferred from their highly divergent allelic constitution. However, in the case of Speonomus, the existence of reproductive barriers was also supported by laboratory experiments (Sbordoni and Menichetti, unpublished; Juberthie-Jupeau, 1988). The occurrence of allopatric sibling species in caves may be due to parallel evolution or similarity in trends of phenotypic changes in populations diverging genetically when subjected to similar selection regimes (Cohan, 1984). Concurrently, divergence at neutral or sligthly deleterious genes, eventually not associated with phenotypic divergence, is often enhanced between small cave population by genetic drift (Sbordoni, 1982).

The present paper deals with the secondary sympatry of two sibling cave shrimps, providing direct evidence of their status as distinct species and arguments in favour of their allopatric origin as well. Sympatric occurrence of sibling cave species has not previously been reported.

Freshwater shrimps of the genus Troglocaris (fam. Atyidae) are among the most representative inhabitants of the subterranean waters in the Karst areas of NE Italy and Yugoslavia. They often occur together with the cave salamander Proteus anguinus and constitute an important part of its diet. The genus Troglocaris shows a disjunct, relict distribution and, according to morphological criteria, it is considered to consist of four species: 1. inermis Fage, 1937, trom Cevennes (Southern France), T. anophthalmus (Kollar, 1848) a polytypic taxon 
occurring in the region NE of the Adriatic Sea (North-Eastern Italy and Yugoslavia), T. hercegovinensis (Babic, 1922) known from Herzegovina and Montenegro (Yugoslavia), and T. kutaissiana (Sadovsky, 1930), also polytypic, from Western Transcaucasia (Georgia, U.S.S.R.) (Holthuis, 1986). A distribution map is reported in Fig. 1.

The Italian populations of $T$. anophthalmus are attributable to the nominate subspecies on the basis of morphological characters (Holthuis, 1986) and are known from a few caves and wells belonging to different drainage systems in the narrow Karst area extending from Gorizia to Trieste (Fig. 2).

Preliminary research on three Italian samples (Allegrucci et al., 1981) suggested the existence of two sibling species, their genetic distinctness indicated by electrophoretically diagnostic alleles at enzyme loci, occurring sympatrically at one locality (Duino Well). The present study is an analysis of most of the known Italian populations in order to assess their taxonomic status, their genetic-geographic relationships, and to inquire whether gene flow exists between this two hypothesized species. In addition, we studied a sample of $T$. inermis from France as an outgroup taxon for evaluating relationships within the genus Troglocaris.

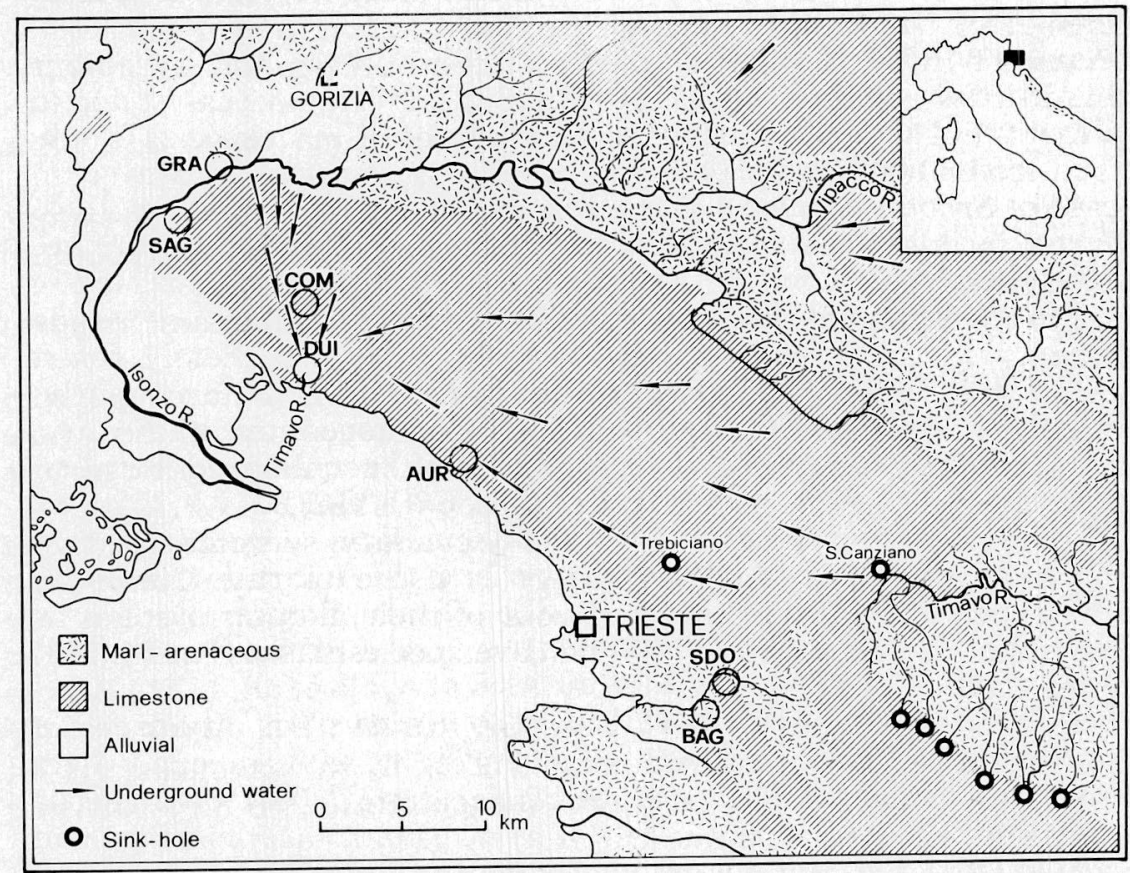

Fig. 2 - Geological out line of the Gorizia-Trieste Karst area indicating main directions of flooding ground waters with collecting sites of Italian Troglocaris: GRA, Frari Well; SAG, Sagrado Cave; COM, Comarie Cave; DUI, Duino Well; AUR, Aurisina Springs; BAG, Bagnoli Cave; SDO, Seasonal Spring near Fonte Oppia. 


\section{MATERIALS AND METHODS}

The Italian samples were collected from three contiguous areas, Gorizia Karst, Trieste Karst and Rosandra Valley. In some of these localities collections were replicated during different water conditions in different years. Samples are characterized by locality symbol, collection site, cave register number, altitude, municipality and province, collection periods and number of specimens assayed, as in the following list.

Gorizia Karst samples:

GRA- Frari Well, VG 4911, m 30 a.s.l., Gradisca di Isonzo (GO); jan. 87; $\mathrm{N}=5$.

SAG- Sagrado Cave, VG 4112, m 25 a.s.l., Sagrado (GO); sept. 80, teb. $85 ; \mathrm{N}=47$.

COM- Comarie Cave, VG 4121, m 15 a.s.l., Doberdò del Lago (GO); dec. 83 , jan. $86 ; \mathrm{N}=83$.

Trieste Karst samples:

DUI- Duino Well, VG 226, m 50 a.s.I., Duino-Aurisina (TS); sept. 80, dec. $83 ; \mathrm{N}=57$.

AUR-Aurisina Springs, $\mathrm{m} 0$ a.s.I., Duino-Aurisina (TS); jan. 87; $\mathrm{N}=12$.

Rosandra Valley samples:

BAG- Bagnoli Cave, VG 105, m 64 a.s.l., San Dorligo della Valle (TS); sept. 80 , nov. 83 , jan. $87 ; \mathrm{N}=49$.

SDO- Seasonal Spring near Fonte Oppia Spring, m 93 a.s.l., San Dorligo della Valle (TS); feb. $87 ; \mathrm{N}=22$.

Location of Italian samples is reported in Fig. 2. The French sample (FRA) of $T$. inermis was collected in the Sauve Cave, Gard, Southern France; oct. $85 ; \mathrm{N}=30$. $-80^{\circ} \mathrm{C}$.

The samples were transported alive to the laboratory and frozen at

Horizontal electrophoresis was performed on $12 \%$ starch gel from Connaught Laboratories by using crude homogenates of the whole body. The following enzymatic proteins have been assayed for genetic variation: ACPH, ADA, AO, APH, CA, EST, GOT, G6PD, LAP, LDH, ME, MPI, PEP, PGM, PHI, TO. The electrophoretic systems used, the staining techniques, the abbreviations and the Enzyme Commission number are detailed in Table 1 . The electrophoretic systems are designed as follow: $\mathrm{A}=$ discontinuous tris citrate, $\mathrm{pH}$ 8.6 (Poulik, 1957); $\mathrm{B}=$ tris versene borate, $\mathrm{pH} 9.1$ (as buffer $\mathrm{B}$ of Ayala et al., 1972); $\mathrm{B}_{1}=$ as buffer $\mathrm{B}$ but with NADP+ added; $\mathrm{C}=$ continuous tris citrate, $\mathrm{pH} 8.4$ (modified from buffer $\mathrm{C}$ of Avala et al., 1972); D = tris maleate, $\mathrm{pH} 7.4$ (Brewer and Sing, 1970); $\mathrm{E}=$ continuous tris citrate, $\mathrm{pH} 8.0$ (Ward and Beardmore, 1977).

Allozymes were numbered relative to the commonest one designated 100 .

The genetic variability of samples was estimated by $\mathrm{H}_{e}$ (expected mean heterozygosity under Hardy-Weinberg equilibrium), $\mathrm{H}_{\mathrm{o}}$ (obser- 


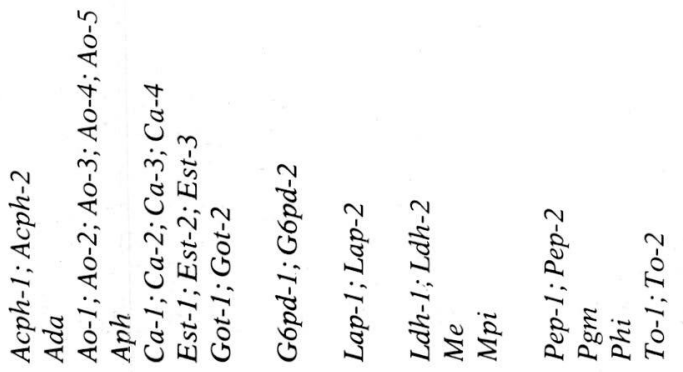

$\stackrel{\infty}{\stackrel{2}{a}}$

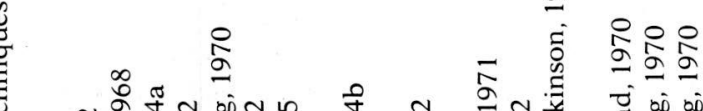

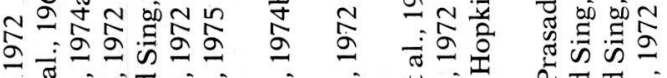

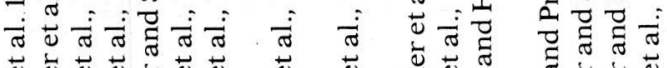

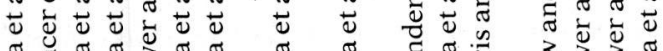

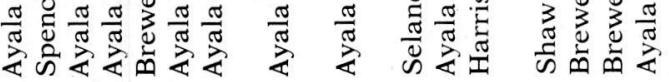

Z

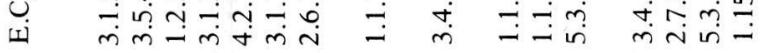


ved mean heterozygosity), $\mathrm{P}$ (proportion of polymorphic loci in according to the criterion of the second most common allele being at least $1 \%$ ), A (mean number of alleles per locus), and $\mathrm{H}^{\prime}$ (Shannon diversity index; Shannon and Weaver, 1963).

The genetic relationships between species and populations were evaluated through the unbiased estimate of standard genetic distance (Nei, 1978).

The dendrogram was drawn on the basis of the $\mathrm{D}$ values, using the UPGMA method of cluster analysis (Sneath and Sokal, 1973).

Factorial analysis of correspondences (Benzecri, 1973) was used to examine in detail allele frequency relationships among samples and genotypes.

\section{RESULTS}

Thirty-two inferred loci were consistently scored. Twenty-seven loci were monomorphic in all samples: Acph-1, Acph-2, Ao-1, Ao-2, Ao-3, Ao-4, Ao-5, Aph, Ca-1, Ca-2, Ca-3, Ca-4, Est-1, Est-2, Est-3, Got-1, Got-2, G6pd-2, Lap-1, Ldh-1, Ldh-2, Me, Mpi, Pep-1, Pep-2, To-1, To-2. The population of Troglocaris inermis (FRA) was monomorphic at all analyzed loci; eighteen loci were found to be diagnostic with the following alternative alleles for this population: Acph-2110, $\mathrm{Ca}-1^{90}$, Ca-2 ${ }^{110}, C a-3^{110}$, Est-1 ${ }^{90}$, Est-2 ${ }^{90}$, Est-390, Got-1 ${ }^{90}$, Got-2 ${ }^{90}$, G6pd-1 ${ }^{90}$, G6pd-2 ${ }^{110}$, Lap-2 ${ }^{90}$, Ldh-2 ${ }^{110}, M e^{110}, M p i^{110}$, Pep- $2^{90}$, Phi ${ }^{90}$, To- ${ }^{90}$.

Among the Italian population five loci were polymorphic, namely: Ada, G6pd-1, Lap-2, Pgm, Phi.

Four loci (Ca-1, Est-1, Me, Pep-2) have been found to be diagnostic for the populations of Bagnoli (BAG) and S. Dorligo (SDO).

At six other loci ( $\mathrm{Ca}-2$, Est-3, Got-2, G6pd-2, Mpi, Phi) homozygous genotypes for each of two alternative alleles have been found to coexist in the samples of Duino (DUI), Sagrado (SAG) and Comarie (COM). With the exception of two individuals from Comarie, showing a heterozygous pattern at the Phi locus, no heterozygote was detected at each of these loci.

Overall the analysis of 275 individuals showed the occurrence of 18 different multilocus genotypes (Table 2).

Results from a correspondence analysis of these multilocus genotypes is shown in Fig. 3. In the diagram the different genotypes split into three groups (A, B, C), in different quadrants within the factorial plane determined by the first two axes, together explaining $80 \%$ of the overall variance. The displacement of the three groups is determined by the alleles exclusive of each group, namely: $C a-2^{100}, E s t-3^{100}$, Got-2 ${ }^{100}$, G6pd-2 ${ }^{95}, \mathrm{Mpi}^{100}, \mathrm{Phi}^{100}$, for group A; Ca-2 ${ }^{95}, E s t-3^{95}, \mathrm{Got}^{295}, \mathrm{G}^{9} p d-2^{100}$, $M p i^{95}, P h i^{95}$, for group B; Ca-1 ${ }^{98}, E s t-3^{98}, M e^{105}, P e p-2^{98}$, for group C, which shares alleles with group B at the loci Ca-2,Est-3, Got-2, G6pd-2, Mpi. While all the genotypes from Bagnoli and S. Dorligo samples 


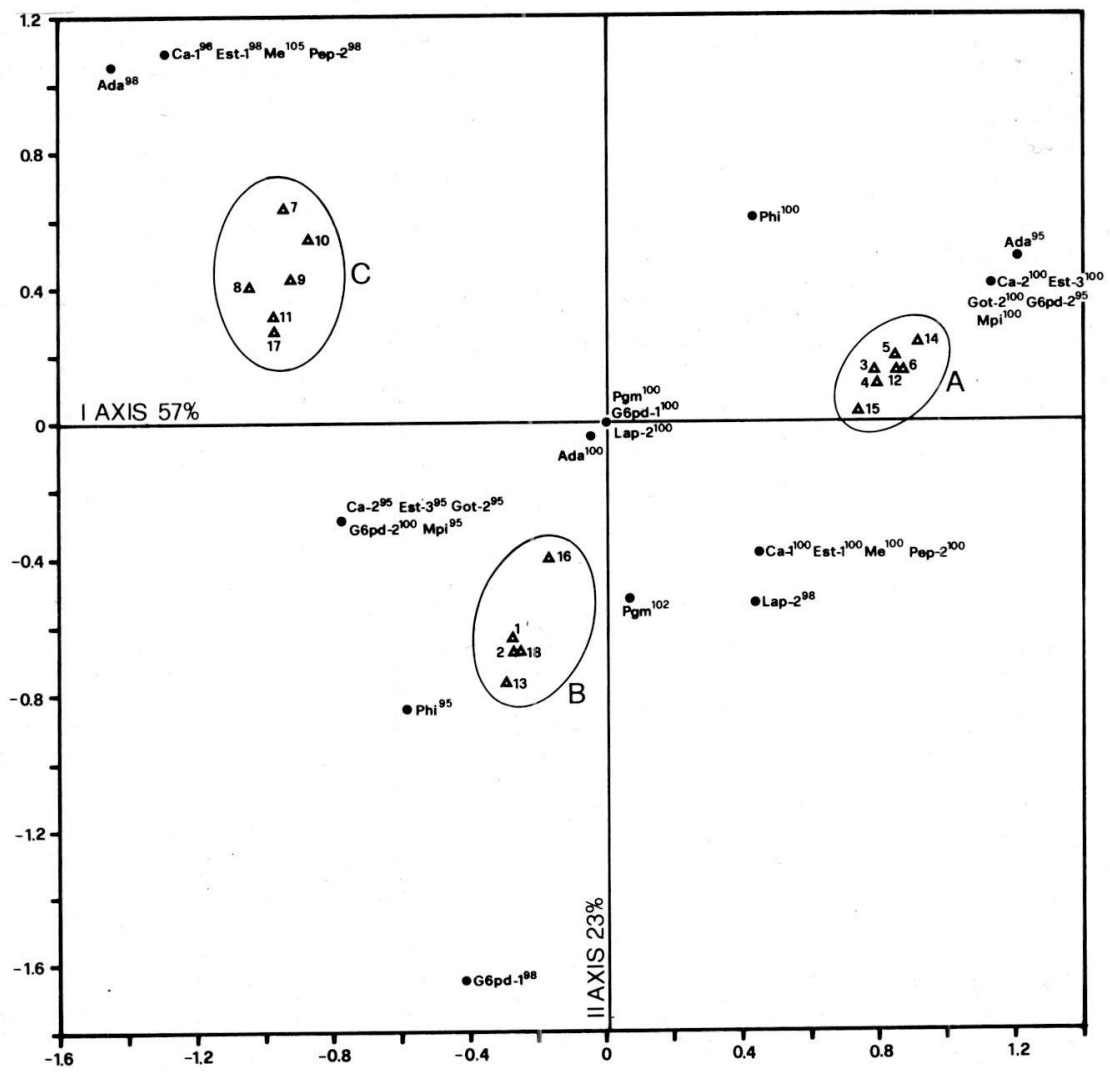

Fig. 3 - A two dimensional plot of 18 different multilocus genotypes of Italian Troglocaris samples resulting from correspondence analysis of 275 individual genotypes. Triangles denote multilocus genotypes, circles denote alleles at enzyme loci.

cluster together in C, most other samples are placed into groups A or B. For example, individuals characterized by genotype $n^{\circ} 1$ of the group $B$ and individuals with the genotype $\mathrm{n}^{\circ} 3$ of the group A occur together in Duino, Sagrado and Comarie localities (Table 2 and Fig. 3).

At these localities the occurrence of fixed allele differences at the six loci, clearly showed the existence of two diversified and isolated groups of genotypes, not only in the samples collected in different years at the Duino Well, but also in the samples collected at Sagrado and Comarie caves. The allele frequencies calculated for the A, B, C groups in the different localities are shown in Table 3.

The correspondence analysis of the allele frequencies displayed evolutionary relationships similar to those shown by multilocus genotypes analysis (Fig. 4).

Samples from Gradisca (GRA) and Aurisina (AUR) are only 


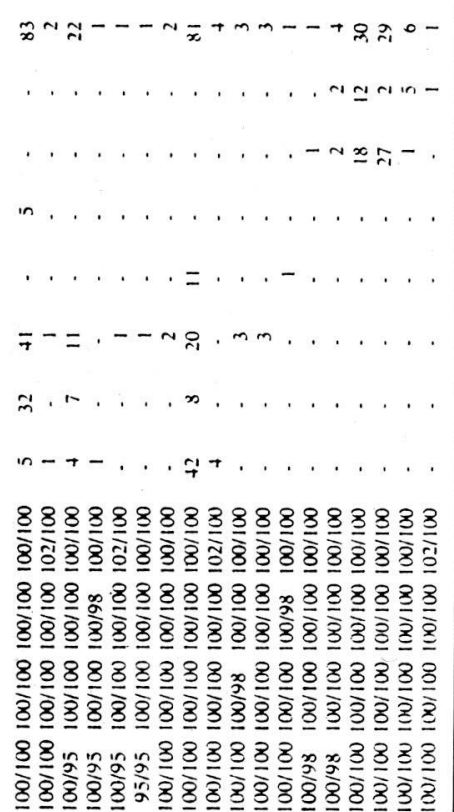

8888 요용요용요

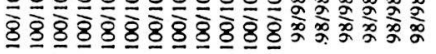

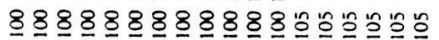

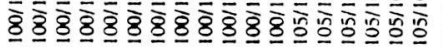

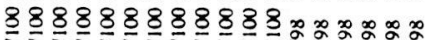

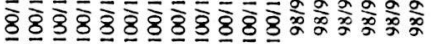
$88888888888 \%$

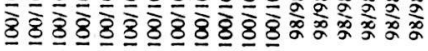
88888 은

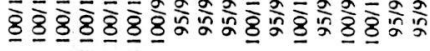

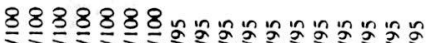

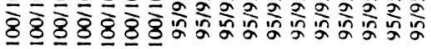

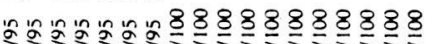

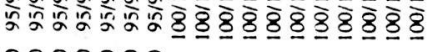

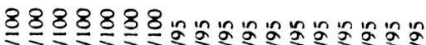

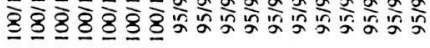

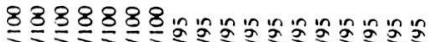

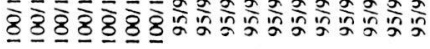

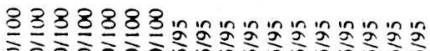

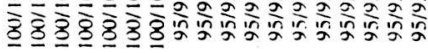


represented by either one or the other of the A-B complex (Table 2 and Fig. 3).

On the basis of the allelic frequency data, genetic identity (I) and standard genetic distance (D) values have been calculated (Table 4), utilizing Nei's (1978) method. D values between the Italian samples confirm the hypothesis of the existence of three groups of populations, provisionally indicated as "species" A, B, C. Genetic distances between populations within each group are extremely low. Much higher values have been revealed between populations belonging to different groups (A vs $\mathrm{B}, \overline{\mathrm{D}}=0.209 ; \mathrm{A}$ vs $\mathrm{C}, \overline{\mathrm{D}}=0.346$; $\mathrm{B}$ vs $\mathrm{C}, \overline{\mathrm{D}}=0.146$ ). The French sample of $T$. inermis appears to be consistently differentiated from each of the Italian populations (average $\mathrm{D}=0.827$ ). The dendrogram of Fig. 5 summarizes genetic distance relationships between populations.

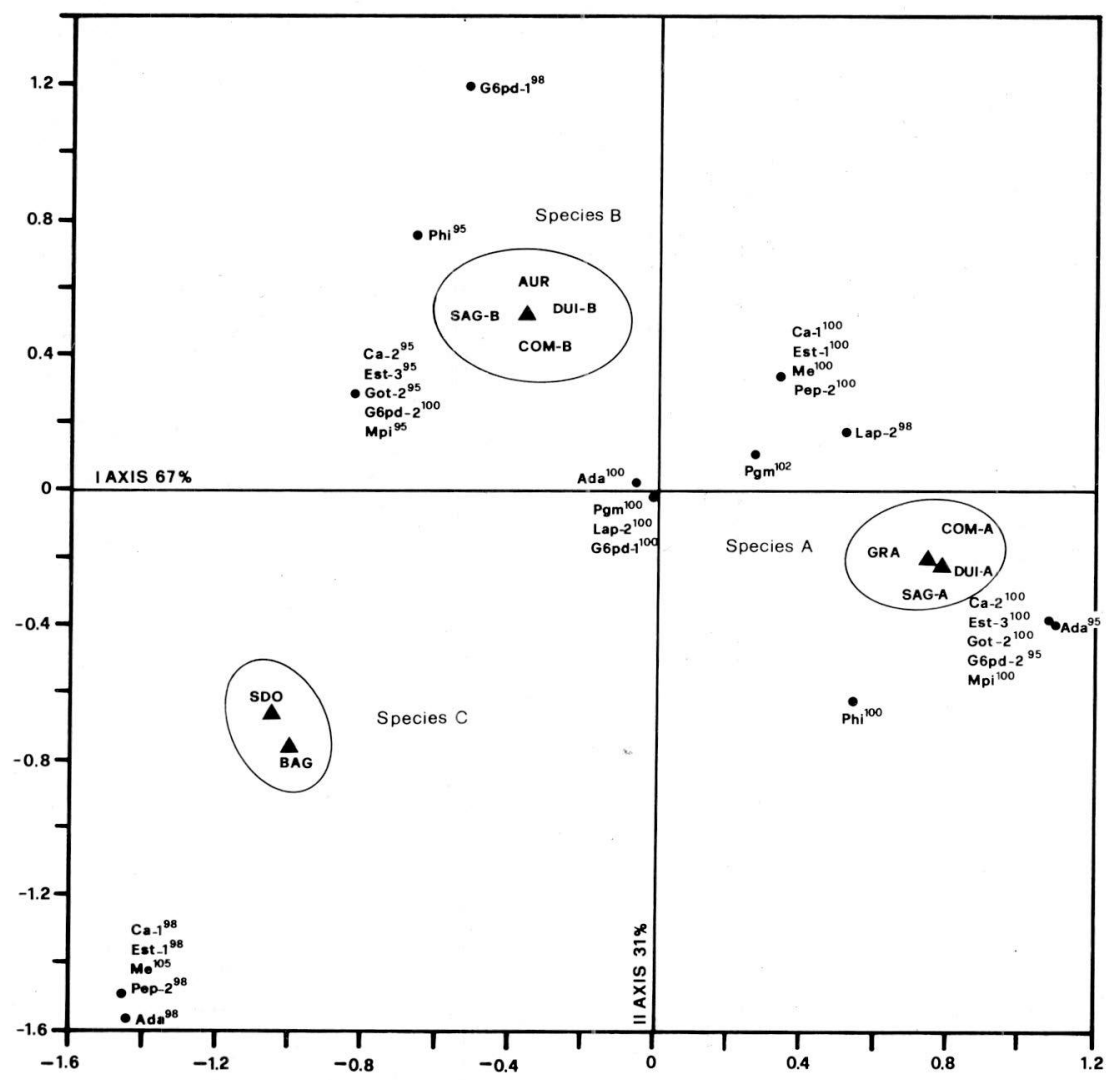

Fig. 4 - A two dimensional plot of populations of Troglocaris anophthalmus complex resulting from correspondence analysis of allele frequencies. Triangles denote populations, circles denote alleles at enzyme loci. 
Table 3 - Allele frequencies of Troglocaris samples. $\mathrm{N}$ = number of individuals assayed.

Locus allele DUI-A DUI-B SAG-A SAG-B BAG COM-A COM-B SDO AUR GRA FRA

$\begin{array}{lllllllllllll}\text { Acph-1 } & 100 & 1 & 1 & 1 & 1 & 1 & 1 & 1 & 1 & 1 & 1 & 1\end{array}$ $\begin{array}{llllllllllll}\mathrm{N} & 11 & 46 & 39 & 8 & 47 & 53 & 30 & 22 & 12 & 5 & 30\end{array}$

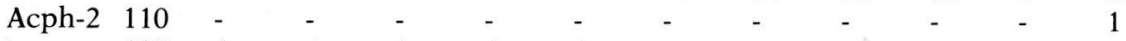

$\begin{array}{llllllllllll}\mathbf{N} & 11 & 46 & 39 & 8 & 47 & 53 & 30 & 22 & 12 & 5 & 30\end{array}$

$\begin{array}{lllllllllllll}\text { Ada } & 100 & 0.75 & 1 & 0.912 & 1 & 0.980 & 0.885 & 1 & 1 & 1 & 1 & 1\end{array}$

$\begin{array}{lllllllllll}98 & - & - & - & - & 0.020 & - & - & - & - & - \\ 95 & 0.250 & - & 0.088 & - & - & 0.115 & - & - & - & -\end{array}$

$\begin{array}{llllllllllll}\mathrm{N} & 4 & 20 & 17 & 5 & 25 & 26 & 9 & 22 & 12 & 5 & 30\end{array}$

$\begin{array}{lllllllllllll}\text { Ao-1 } & 100 & 1 & 1 & 1 & 1 & 1 & 1 & 1 & 1 & 1 & 1 & 1\end{array}$

$\begin{array}{lllllllllllll} & \mathrm{N} & 11 & 46 & 39 & 8 & 47 & 53 & 30 & 22 & 12 & 5 & 30 \\ \text { Ao-2 } & 100 & 1 & 1 & 1 & 1 & 1 & 1 & 1 & 1 & 1 & 1 & 1\end{array}$

$\begin{array}{lllllllllllll} & \mathrm{N} & 11 & 46 & 39 & 8 & 47 & 53 & 30 & 22 & 12 & 5 & 30\end{array}$

$\begin{array}{lllllllllllll}\text { Ao-3 } & 100 & 1 & 1 & 1 & 1 & 1 & 1 & 1 & 1 & 1 & 1 & 1\end{array}$

$\begin{array}{lllllllllllll} & \mathrm{N} & 11 & 46 & 39 & 8 & 47 & 53 & 30 & 22 & 12 & 5 & 30\end{array}$

$\begin{array}{llllllllllll}\mathrm{N} & 11 & 46 & 39 & 8 & 1 & 1 & 1 & 1 & 1 & 1 & 1 \\ \mathrm{~A} & 11 & 4 & 53 & 30 & 22 & 12 & 5 & 30\end{array}$

$\begin{array}{lllllllllllll}\text { Ao-5 } & 100 & 1 & 1 & 1 & 1 & 1 & 1 & 1 & 1 & 1 & 1 & 1\end{array}$

$\begin{array}{lllllllllllll} & \mathrm{N} & 11 & 46 & 39 & 8 & 47 & 53 & 30 & 22 & 12 & 5 & 30 \\ \text { Aph } & 100 & 1 & 1 & 1 & 1 & 1 & 1 & 1 & 1 & 1 & 1 & 1\end{array}$

$\begin{array}{lllllllllllll} & \mathbf{N} & 11 & 46 & 39 & 8 & 47 & 53 & 30 & 22 & 12 & 5 & 30\end{array}$

$\begin{array}{llllllllllll}98 & - & - & - & - & 1 & - & - & 1 & - & - & - \\ 90 & - & - & - & - & - & - & - & - & - & - & 1 \\ \text { N } & 11 & 46 & 39 & 8 & 47 & 53 & 30 & 22 & 12 & 5 & 30\end{array}$

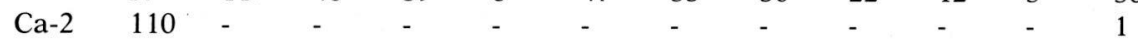

$\begin{array}{llllllllll}100 & 1 & - & 1 & - & - & 1 & - & - & -\end{array} 1$

$\begin{array}{lllllllllllll} & 95 & - & 1 & - & 1 & 1 & - & 1 & 1 & 1 & - & - \\ \mathrm{Ca}-3 & \mathrm{~N} & 11 & 46 & 39 & 8 & 47 & 53 & 30 & 22 & 12 & 5 & 30\end{array}$

$\begin{array}{lllllllllllll} & 100 & 1 & 1 & 1 & 1 & 1 & 1 & 1 & 1 & 1 & 1 & - \\ \mathrm{Ca}-4 & \mathrm{~N} & 11 & 46 & 39 & 8 & 47 & 53 & 30 & 22 & 12 & 5 & 30 \\ & 100 & 1 & 1 & 1 & 1 & 1 & 1 & 1 & 1 & 1 & 1 & 1 \\ & \mathrm{~N} & 11 & 46 & 39 & 8 & 47 & 53 & 30 & 22 & 12 & 5 & 30\end{array}$

$\begin{array}{lllllllllllll}\text { Est-1 } & 100 & 1 & 1 & 1 & 1 & - & 1 & 1 & - & 1 & 1 & - \\ & 98 & - & - & - & - & 1 & - & - & 1 & - & - & -\end{array}$

$\begin{array}{llllllllllll}\mathrm{N} & 11 & 46 & 39 & 8 & 47 & 53 & 30 & 22 & 12 & 5 & 30\end{array}$

$\begin{array}{lllllllllllll}\text { Est-2 } & 100 & 1 & 1 & 1 & 1 & 1 & 1 & 1 & 1 & 1 & 1 & -\end{array}$

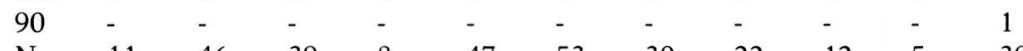

$\begin{array}{lllllllllllll} & \mathrm{N} & 11 & 46 & 39 & 8 & 47 & 53 & 30 & 22 & 12 & 5 & 30 \\ \text { Est-3 } & 100 & 1 & - & 1 & - & - & 1 & - & - & - & 1 & -\end{array}$

$\begin{array}{llllllllllll}90 & - & - & - & - & - & - & - & - & - & - & 1 \\ \mathrm{~N} & 11 & 46 & 39 & 8 & 47 & 53 & 30 & 22 & 12 & 5 & 30\end{array}$

$\begin{array}{lllllllllllll}\text { Got-1 } & 100 & 1 & 1 & 1 & 1 & 1 & 1 & 1 & 1 & 1 & 1 & -\end{array}$ $\begin{array}{llllllllllll}90 & - & - & - & - & - & - & - & - & - & - & 1 \\ \mathrm{~N} & 11 & 46 & 39 & 8 & 47 & 53 & 30 & 22 & 12 & 5 & 30\end{array}$ 
Locus allele DUI-A DUI-B SAG-A SAG-B BAG COM-A COM-B SDO AUR GRA FRA

Got-2 $100 \quad 1 \quad-\quad 1$

$$
\begin{array}{llllllllllll}
95 & - & 1 & - & 1 & 1 & - & 1 & 1 & 1 & - & - \\
90 & - & - & - & - & - & - & - & - & - & - & 1 \\
N & 11 & 46 & 39 & 8 & 47 & 53 & 30 & 22 & 12 & 5 & 30
\end{array}
$$

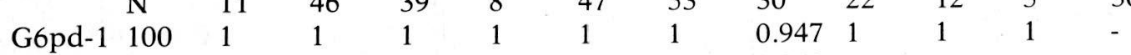

$$
\begin{array}{lllllllllll}
100 & - & - & - & - & - & - & 0.053- & - & - & - \\
90 & - & - & - & - & - & - & - & - & - & -
\end{array}
$$$$
\begin{array}{llllllllllll}
\mathrm{N} & 11 & 46 & 39 & 8 & 47 & 40 & 19 & 22 & 12 & 5 & 30
\end{array}
$$

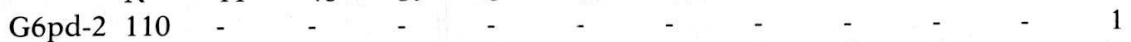

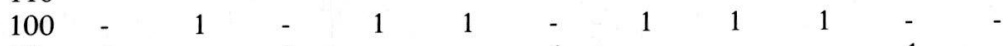
$\begin{array}{llllllllllll}95 & 1 & - & 1 & - & - & 1 & - & - & - & 1 & -\end{array}$

$\begin{array}{lllllllllllll}\text { Lap-1 } & 100 & 1 & 1 & 1 & 1 & 1 & 1 & 1 & 1 & 1 & 1 & 1\end{array}$
$\begin{array}{llllllllllll}\mathbf{N} & 11 & 46 & 39 & 8 & 47 & 53 & 30 & 22 & 12 & 5 & 30\end{array}$ $\begin{array}{lllllllllllll}\text { Lap-2 } & 100 & 1 & 1 & 1 & 1 & 1 & 1 & 1 & 1 & 0.929 & 1 & - \\ & 98 & - & - & - & - & - & - & - & - & 0.071 & - & -\end{array}$

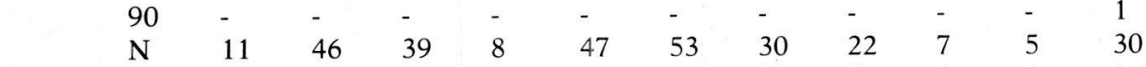

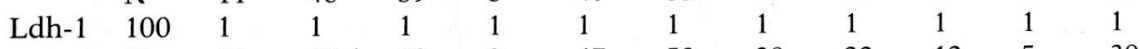

\begin{tabular}{|c|c|c|c|c|c|c|c|c|c|c|c|c|}
\hline \multirow[t]{4}{*}{$\mathrm{Me}$} & 110 & - & - & - & - & - & - & - & - & - & - & 1 \\
\hline & 105 & - & - & - & - & 1 & - & - & 1 & - & - & - \\
\hline & 100 & 1 & 1 & 1 & 1 & - & 1 & 1 & - & 1 & 1 & - \\
\hline & $\mathrm{N}$ & 11 & 46 & 39 & 8 & 47 & 53 & 30 & 22 & 12 & 5 & 30 \\
\hline \multirow[t]{4}{*}{ Mpi } & 110 & - & - & - & - & - & - & - & - & - & - & 1 \\
\hline & 100 & 1 & - & 1 & - & - & 1 & - & - & - & 1 & - \\
\hline & 95 & - & 1 & - & 1 & 1 & - & 1 & 1 & 1 & - & - \\
\hline & $\mathrm{N}$ & 11 & 46 & 39 & 8 & 47 & 53 & 30 & 22 & 12 & 5 & 30 \\
\hline \multirow[t]{2}{*}{ Pep-1 } & 100 & 1 & 1 & 1 & 1 & 1 & 1 & 1 & 1 & 1 & 1 & 1 \\
\hline & $\mathrm{N}$ & 11 & 46 & 39 & 8 & 47 & 53 & 30 & 22 & 12 & 5 & 30 \\
\hline \multirow[t]{4}{*}{ Pep-2 } & 100 & 1 & 1 & 1 & 1 & - & 1 & 1 & - & 1 & 1 & - \\
\hline & 98 & - & - & - & - & 1 & - & - & 1 & - & - & - \\
\hline & 90 & - & - & - & - & - & - & - & - & - & - & 1 \\
\hline & $\mathrm{N}$ & 11 & 46 & 39 & 8 & 47 & 53 & 30 & 22 & 12 & 5 & 30 \\
\hline \multirow[t]{3}{*}{ Pgm } & 102 & 0.045 & 0.053 & - & - & - & 0.023 & - & 0.024 & - & - & - \\
\hline & 100 & 0.955 & 0.947 & 1 & 1 & 1 & 0.977 & 1 & 0.976 & 1 & 1 & 1 \\
\hline & $\mathrm{N}$ & 11 & 38 & 37 & 5 & 43 & 43 & 22 & 21 & 4 & 5 & 30 \\
\hline \multirow[t]{4}{*}{ Phi } & 100 & 1 & - & 1 & - & 0.762 & 0.976 & - & 0.353 & - & 1 & - \\
\hline & 95 & - & 1 & - & 1 & 0.238 & 0.024 & 1 & 0.647 & 1 & - & - \\
\hline & 90 & - & - & - & - & - & - & - & - & - & - & 1 \\
\hline & $\mathrm{N}$ & 11 & 46 & 39 & 8 & 42 & 84 & 38 & 17 & 12 & 5 & 30 \\
\hline \multirow[t]{2}{*}{ To-1 } & 100 & 1 & 1 & 1 & 1 & 1 & 1 & 1 & 1 & 1 & 1 & 1 \\
\hline & $\mathrm{N}$ & 11 & 46 & 39 & 8 & 47 & 53 & 30 & 22 & 12 & 5 & 30 \\
\hline \multirow[t]{3}{*}{ To-2 } & 100 & 1 & 1 & 1 & 1 & 1 & 1 & 1 & 1 & 1 & 1 & - \\
\hline & 90 & - & - & - & - & - & - & - & - & - & - & 1 \\
\hline & $\mathrm{N}$ & 11 & 46 & 39 & 8 & 47 & 53 & 30 & 22 & 12 & 5 & 30 \\
\hline
\end{tabular}

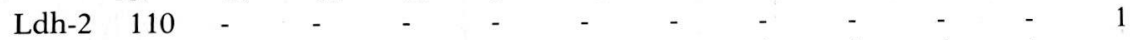
$\begin{array}{llllllllllll}100 & 1 & 1 & 1 & 1 & 1 & 1 & 1 & 1 & 1 & 1 & - \\ \mathrm{N} & 11 & 46 & 39 & 8 & 47 & 53 & 30 & 22 & 12 & 5 & 30\end{array}$ 
Table 4 - Genetic identity (above) and genetic distance (below) values between populations and species of Troglocaris.

DUI-A DUI-B SAG-A SAG-B BAG COM-A COM-B SDO AUR GRA FRA

\begin{tabular}{|c|c|c|c|c|c|c|c|c|c|c|c|}
\hline DUI-A & $* * * * *$ & 0.810 & 1.000 & 0.810 & 0.712 & 1.000 & $0.810^{\circ}$ & 0.700 & 0.809 & 0.999 & 0.432 \\
\hline DUI-B & 0.211 & $* * * * *$ & 0.811 & 1.000 & 0.856 & 0.812 & 1.000 & 0.870 & 1.000 & 0.812 & 0.437 \\
\hline SAG-A & 0.000 & 0.209 & $* * * * *$ & 0.812 & 0.714 & 1.000 & 0.811 & 0.703 & 0.811 & 1.000 & 0.436 \\
\hline SAG-B & 0.211 & 0.000 & 0.208 & $* * * * *$ & 0.856 & 0.813 & 1.000 & 0.870 & 1.000 & 0.812 & 0.437 \\
\hline BAG & 0.340 & 0.156 & 0.336 & 0.155 & $\star \star \star \star \star \star$ & 0.714 & 0.856 & 0.995 & 0.856 & 0.715 & 0.440 \\
\hline COM-A & 0.000 & 0.208 & 0.000 & 0.207 & 0.337 & 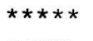 & 0.812 & 0.703 & 0.812 & 1.000 & 0.435 \\
\hline COM-B & 0.211 & 0.000 & 0.209 & 0.000 & 0.156 & 0.208 & $* * * * *$ & 0.870 & 1.000 & 0.812 & 0.438 \\
\hline SDO & 0.357 & 0.139 & 0.353 & 0.139 & 0.005 & 0.353 & 0.139 & $* * * * *$ & 0.870 & 0.704 & 0.440 \\
\hline AUR & 0.211 & 0.000 & 0.209 & 0.000 & 0.156 & 0.208 & 0.000 & 0.139 & $\star \star \star \star \star *$ & 0.812 & 0.438 \\
\hline GRA & 0.001 & 0.208 & 0.000 & 0.208 & 0.335 & 0.000 & 0.208 & 0.352 & 0.208 & 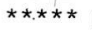 & 0.437 \\
\hline FRA & 0.840 & 0.829 & 0.830 & 0.827 & 0.822 & 0.832 & 0.825 & 0.820 & 0.824 & 0.827 & $\star * \star * * *$ \\
\hline
\end{tabular}

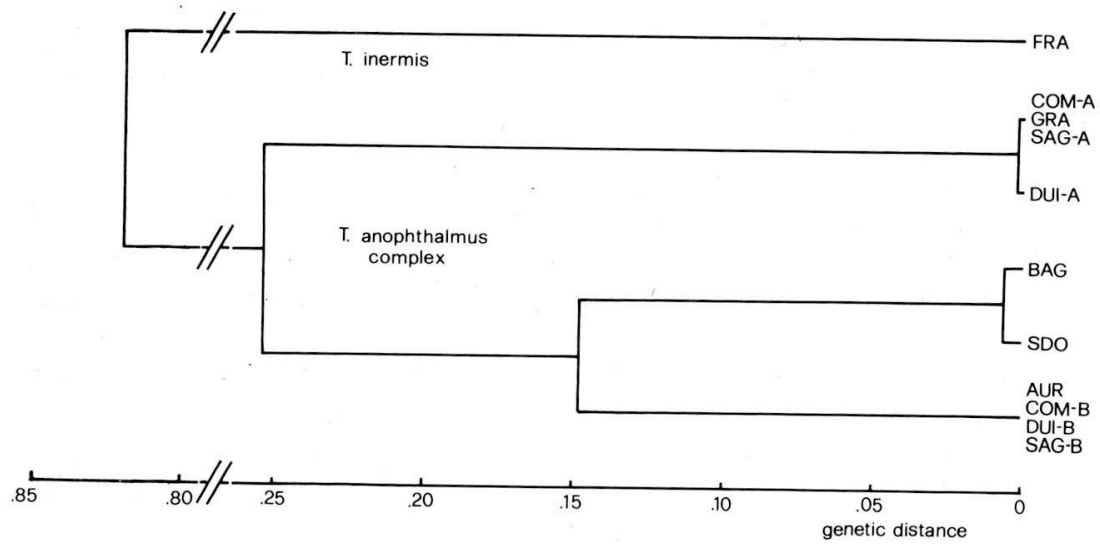

Fig. 5 - UPGMA dendrogram showing genetic relationships between populations and species of Troglocaris.

Table 5 gives the genetic variability estimates for all studied populations of Troglocaris. All estimates of observed $\left(\mathrm{H}_{\mathrm{o}}\right)$, and expected $\left(\mathrm{H}_{\mathrm{e}}\right)$ heterozygosity, and the Shannon index $\left(\mathrm{H}^{\prime}\right)$ indicate an unusually low level of genetic polymorphism: $\mathrm{H}_{\mathrm{e}}$ ranges from 0 to 0.023 among the Italian populations, with the French population being monomorphic. 
Table 5 - Variability estimates of Troglocaris populations

\begin{tabular}{|c|c|c|c|c|c|c|c|}
\hline & Species & Population & $\mathrm{N}$ & Ho & $\mathrm{He}$ & $\begin{array}{l}\text { not recorded } \\
\text { multicolus } \\
\text { genotypes }\end{array}$ & $\begin{array}{l}\text { genotypic diversity } \\
\text { index } H^{\prime} \text { (Shannon) }\end{array}$ \\
\hline T. inermis & & FRA & 30 & 0.000 & 0.000 & 1 & 0.000 \\
\hline \multirow{10}{*}{$\begin{array}{l}T \text {. anophthalmus } \\
\text { complex }\end{array}$} & \multirow[t]{4}{*}{ A } & DUI & 11 & 0.029 & 0.023 & 4 & 0.504 \\
\hline & & SAG & 39 & 0.006 & 0.004 & 2 & 0.204 \\
\hline & & COM & 57 & 0.014 & 0.012 & 6 & 0.384 \\
\hline & & GRA & 5 & 0.000 & 0.000 & 1 & 0.000 \\
\hline & \multirow[t]{4}{*}{ B } & DUI & 46 & 0.003 & 0.003 & 2 & 0.128 \\
\hline & & SAG & 8 & 0.000 & 0.000 & 1 & 0.000 \\
\hline & & COM & 26 & 0.004 & 0.004 & 3 & 0.304 \\
\hline & & AUR & 12 & 0.004 & 0.004 & 2 & 0.124 \\
\hline & \multirow[t]{2}{*}{ C } & BAG & 48 & 0.016 & 0.015 & 5 & 0.428 \\
\hline & & SDO & 22 & 0.020 & 0.018 & 5 & 0.540 \\
\hline
\end{tabular}

\section{DISCUSSION}

Results from the analysis of allozyme genotypes and allele frequencies have clearly shown the existence of separate biological species in the samples collected in different years at the Duino Well (DUI), Sagrado (SAG) and Comarie (COM) caves. Two species, provisionally indicated as species $\mathrm{A}$ and $\mathrm{B}$ in the $T$. anophthalmus complex, appear to coexist syntopically in these three localities. Indeed, in spite of their sympatric occurrence no heterozygote was detected at any of the loci fixed for alternative alleles (with the exception of the locus Phi of COM A sample), indicating a lack of gene flow between these species. Two individuals have been found with an heterozygous pattern at the $P h i$ locus in the COM sample; they have been identified as species A on the basis of their remaining five diagnostic loci. This outcome might well be the result of an introgression. Nevertheless it could also be explained as a polymorphism at the Phi locus, taking into account that a similar electrophoretic mobility of allozymatic forms might mask substantial differences. In this way the $P h^{95}$ allele of the two COM individuals may be different from $\mathrm{Phi}^{95}$ allele of species A. Similar features may count also for $P h i^{\mathbf{1 0 0}}$ allele of species $\mathrm{C}$ and $\mathrm{A}$. In the ligth of these remarks no clear evidence of any gene flow between species A and species B is available.

Samples from COM, DUI and SAG have been examined to detect any morphological discontinuity at relevant taxonomic features. Since 
careful morphological inspection of the characters usually utilized in the taxonomy of this group did not reveal any character available to separate the two species they can be regarded as true siblings. However, due to the difficulty of collecting adeguate samples, multivariate morphometric analysis has not yet carried out. At present, the assignment of specimens to either species A or B is possible only by means of their electrophoretic patterns. On this basis it has been pointed out that species A is also present in the Gradisca Cave, while the sample collected at the Aurisina Springs belongs to species B. The two remaining population samples from the Rosandra River basin, BAG and SDO, proved to be monospecific too. Because "species C" does not occur sympatrically with A or B, we cannot be as certain of its status as a distinct species. However, the fact that it is fixed for alternative alleles at several loci strongly suggests it is reproductively isolated from A and B as well. This interpretation of data is supported by the analysis of dendrogram (Fig. 5) and the results of the correspondence analysis on allele frequencies (Fig. 4).

An interesting feature of the genetic structure of Troglocaris populations is that genetic distance between samples, within each of the two species $A$ and $B$, are very small $(D \leq 0.001)$ in spite of their relatively high geographic distance (see Fig. 2). Indeed these findings strongly suggest that these various samples actually belong to single panmittic populations both in the species A and in the species B as well.

Similar situations have been found in other stygobiont animals as Spelaeomysis (Crustacea, Mysidacea: De Matthaeis et al., 1982), Typhlocirolana (Crustacea, Isopoda: Caccone et al., 1986), Phreatichthys and Barbopsis (Pisces, Cyprinidae: Cobolli Sbordoni et. al., 1989). On the whole these results show that the genetic structure of several stygobiont organisms is largely affected by the 'continuity' of the underground water systems. Different situations have been often observed in conspecific populations of terrestrial Arthropod cave species due to the prevailing discontinuity of the terrestrial cave biota (Delay et al., 1980; Sbordoni et al., 1985; Crouau-Roy, 1987; Barr and Holsinger, 1985).

The interspecific values of genetic distances in pairwise comparison between the three sibling species range from 0.139 to 0.357 . Similar values of genetic distances have been recorded in other cave species. For example D values range from 0.15 to 0.23 (Sbordoni et al., 1985) in pairwise comparisons between different species of cave Orthoptera belonging to the genus Dolichopoda. Such species proved to be reproductively isolated by postmating barriers in natural and experimental conditions (Allegrucci et al., 1982).

The occurrence of allopatric genetic divergence in subterranean organisms in the absence of consistent morphological differentiation is quite common (Sbordoni, 1982). The potential existence of undiscovered sibling species complexes in several Decapod Crustaceans is emphasized by Knowlton (1986). However, the sympatric occurrence of sibling cave species is an unexpected evolutionary outcome. It has not previously been reported and deserves some comments. 
First, there is a lack of any reasonable basis to hypothesize sympatric speciation leading to adaptive divergence of species A and B. Indeed, repeated visits to Troglocaris caves did not provide any evidence of ecological displacement between the two species. On the contrary they seem to share the same habitat and the same food resources as well. In our opinion all three species in the $T$. anophthalmus complex originated in allopatry. The range of species A and B do not overlap completely, suggesting that species A originated in the underground waters of the Vipacco-Isonzo basin and species B originated in isolation in the Timavo system. Presently, in flooding periods, subterranean waters from the two systems join to various degreees in a rather wide area including the DUI, SAG and COM caves (Fig. 2). Repeated sampling in the three caves in dry periods and during floods yielded significantly different proportions of the two species, suggesting their possible role as markers of flooding ground waters (Table 6).

Table 6 - Number of individuals and relative frequency of Troglocaris species A and species B occurring in successive samples in three caves in the Karst.

\begin{tabular}{|c|c|c|c|c|c|c|}
\hline \multirow[t]{2}{*}{ Cave } & \multirow[t]{2}{*}{$\begin{array}{l}\text { Sampling } \\
\text { date }\end{array}$} & \multirow[t]{2}{*}{$\begin{array}{c}\text { Underground } \\
\text { water level }\end{array}$} & \multicolumn{2}{|c|}{$\begin{array}{l}\text { Individuals and relative } \\
\text { trequency (in brackets) of }\end{array}$} & & \\
\hline & & & species A & species B & & \\
\hline DUI & $\begin{array}{l}\text { Sep } 1980 \\
\text { Dec } 1983\end{array}$ & $\begin{array}{l}\text { dry } \\
\text { dry }\end{array}$ & $\begin{array}{l}7(0.28) \\
4(0.12)\end{array}$ & $\begin{array}{l}18(0.72) \\
28(0.87)\end{array}$ & $x^{2}=1.284$ & n.s. \\
\hline SAG & $\begin{array}{l}\text { Sep } 1980 \\
\text { Feb } 1985\end{array}$ & $\begin{array}{l}\text { dry } \\
\text { flood }\end{array}$ & $\begin{array}{l}20(1.00) \\
19(0.70)\end{array}$ & $\begin{array}{l}0(0.00) \\
8(0.30)\end{array}$ & $x^{2}=5.198$ & $0.01<\mathrm{P}<0.025$ \\
\hline $\mathrm{COM}$ & $\begin{array}{l}\text { Jan } 1983 \\
\text { Jan } 1986\end{array}$ & $\begin{array}{l}\text { dry } \\
\text { flood }\end{array}$ & $\begin{array}{r}7(0.32) \\
77(0.82)\end{array}$ & $\begin{array}{l}15(0.68) \\
17(0.18)\end{array}$ & $x^{2}=19.960$ & $\mathrm{P}<0.005$ \\
\hline
\end{tabular}

In the past, however, complete isolation of these drainage systems is indicated both by tectonic events, wich occurred from 5.2 million years up to 0.7 Myr B.P., and by variations in the underground water level (Carulli et al., 1980). Such geological dating of palaeotectonic events is in agreement with rough estimates of divergence times obtained from allozyme data, by assuming the molecular clock hypothesis. We utilized the relation between genetic distance and absolute time suggested by Nei (1975), $\mathrm{T}=5 \times 10^{6} \times \mathrm{D}$ years, which for low D values require no adjustment for "slow" and "fast" evolving loci. Even if these estimates are subjected to large standard errors associated with the $\mathrm{D}$ values, they provide a good basis for dating allopatric cladogenetic events in "island like" vicariant species (Sbordoni et al., 1990).

According to these estimates speciation events occurred in the $T$. 
anophthalmus complex between 1.7 Myr (species A vs C) and $0.7 \mathrm{Myr}$ B.P. (species B vs C). Presently geographic barriers between species A and $B$ are no long at work. The almost complete lack of geographic variation between populations within each of the two species is probably due to the present high interconnectivity of ground waters but also to the very low level of genetic diversity detected within populations (Table 5).

Another point deserving some comments is the low degree of genetic variability revealed by these Troglocaris shrimps. Levels of heterozygosity recorded in all samples rank these organisms among the less genetically variable cave dwelling species of Crustaceans (Caccone et al., 1986). We hypothesize three explanations, which might account for this outcome:

1) Marginal populations. The different populations of $T$. anophthalmus examined in this study come from the margin of the distribution of the species complex. In some cases (Highton and Webster, 1976; Barker and Mulley, 1976; Prakash et al., 1969) a reduction in allelic polymorphism in peripheral situation has been reported. This hypothesis might be tested through the analysis of Slovenian populations which are in the centre of the range.

2) Genetic variability levels in Crustacea Decapoda. With few exceptions, species of Decapod Crustaceans have been found to be scarcely polymorphic at allozymic loci, and clearly less than other groups of invertebrates (Nelson and Hedgecock, 1980; Nevo et al., 1984). Thus, the observed levels of enzyme polymorphism could simply reflect a particular taxon-dependent genetic pattern.

3) Age of colonization of cave environment. From the results of previous studies on cave organisms belonging to different taxa, genetic variability in cave populations appears to be positively related to evolutionary time (i.e. the time since the last bottleneck). In particular, high values of heterozygosity have been reported in troglobitic species, mostly in aquatic Amphipodan and Isopodan Crustaceans, Niphargus and Monolistra species (Sbordoni et al., 1979; 1980). These cases have been explained as the result of an ancient colonization of hypogean habitats. On the other hand, low genetic variability levels have been found in comparatively more recent invaders of subterranean waters such as Spelaeomysis (De Matthaeis et al., 1982) and Typhlocirolana (Caccone et al., 1986). These Crustaceans belong to the so called "thalassoid" group of organisms, and are thought to have colonized underground water systems during the Pliocene regression of the Mediterranean Sea (Stock, 1967). Low genetic variability of Troglocaris species fits well with this scenario since their cave evolution seems to be relatively recent (Bouvier, 1925). In the Dinaric area, cave colonization has been dated to the end of Pliocene (Sket, 1971) and a few Troglocaris anophthalmus populations still retain "epigean" features such as well developed eyes (Sket, 1985).

These three hypotheses are not mutually exclusive. A fourth hypothesis has sometimes been raised to explain assumed or observed 
low genetic variability in cave animals, i.e., the role of a small effective population size in decreasing variability via inbreeding and genetic drift (Avise and Selander, 1972). This hypothesis seems not to apply to our situation because the lack of any evidence of reduced population size of Troglocaris populations. In addition it has been empirically proved that even very small cave populations, estimated by markrecapture methods, may show high levels of genetic variability (Sbordoni et al., 1987).

On the contrary the great genetic identity revealed between geographically distant populations within a drainage system, seems to account for the existence of a hidden large subterranean shrimp population.

\section{ACKNOWLEDGMENTS}

We thank Fulvio Gasparo for his invaluable help in the collection of samples and for many useful informations on the Karst area. We also acnowledge G. Allegrucci, A. Caccone, and D. Cesaroni for their cooperation in the first part of the studv, F. Baldari for taxonomic inspection, M. Oliverio for technical help, C. Juberthie, L. Juberthie-Jupeau and E. Pickl for help in the collection of samples. The manuscript benefitted by comments of J. Powell and two anonymous referees.

\section{REFERENCES}

ALlEGRUCCI, G., A. CACCONE, D. CESARONI, M. COBOLLI SBORDONI, E. DE MATTHAEIS, and V. SBORDONI. 1981. Prime ricerche sulla genetica di popolazioni del gamberetto cavernicolo Troglocaris anophthalmus. Boll. Zool. 48:12.

ALLEGRUCCI, G., A. CACCONE, D. CESARONI, M. COBOLLI SBORDONI, E. DE MATTHAEIS, and V. SBORDONI. 1982. Natural and experimental interspecific hybridation between populations of Dolichopoda cave crickets. Experientia 38: 96-98.

AVISE, J. C. and R. K. SELANDER. 1972. Evolutionary genetics of cave-dwelling fishes of the genus Astyanax. Evolution 26 (1): 1-19.

AYALA, F. J., J. R. POWELL, M. L. TRACEY, C. A.MOURAO, and S. PEREZ-SALAS. 1972. Enzyme variability in the Drosophila willistoni group. IV. Genetic variation in natural populations of Drosophila willistoni. Genetics 70: 113-139.

AYALA, F. J., M. L. TRACEY, L. G. BARR, and J. G. EHRENFELD. 1974a. Genetic and reproductive differentiation of the subspecies Drosophila equinoxialis caribbensis. Evolution 28: 24-41.

AYALA, F. J., J. W. VALENTINE, L. G. BARR, and G. S. ZUMWALT. 1974b. Genetic variability in a temperate intertidal phoronid, Phoronopsis viridis. Biochem. Genet. 11: 413-427.

AYALA, F. J., J. W. VALENTINE, and G. S. ZUMWALT. 1975. An electrophoretic study of the Antartic zooplankter Euphasia superba. Limnol. Oceanogr. 20: 635-640.

BARKER, J. S. F. and J. C. MULLEY. 1976. Isozyme variation in natural populations of Drosophila buzzatii. Evolution 30: 213-233.

BARR, T.C. 1968. Cave ecology and the evolution of troglobites. Evol. Biol. 2: 35-102.

BARR, T. C. jr., and J. R. HOLSINGER. 1985. Speciation in cave faunas. Ann. Rev. Ecol. Syst. 16: 313-337.

BENZECRI, J. P. 1973. L'Analyse des données. II. Dunod, Paris. 
BOUVIER, E. L. 1925. Recherches sur la morphologie, les variations, la distribution geografique des Crevettes de la famille des Atyides. Encycl. ent. (A) 4: 1-716.

BREWER, G. J. and C. F. SING. 1970. An introduction to isozyme techniques. Academic Press, N.Y.

CACCONE, A., G. ALlEGRUCCI, D. CESARONI, M. COBOLli SBORDONI, E. DE MATTHAEIS, G. LA ROSA, and V. SBORDONI. 1986. Genetic variability and divergence between cave dwelling populations of Typhlocirolana from Majorca and Sicily. Biochemical Systematics and Ecology 14: 215-221.

CARULli, G. B., L. CAROBENE, A. CAVALLIN, B. MARTINIS, and R. ONOFRI. 1980. Evoluzione strutturale plio-quaternaria del Friuli e della Venezia Giulia. In: Contributi alla realizzazione della Carta Neotettonica d'Italia, Parte II (Pubb. n. 356 P.F. Geodinamica) p. 489. CNR, Napoli.

COBOLLI SBORDONI, M., E. DE MATTHAEIS, M. MATTOCCIA, R. BERTI, and V. SBORDONI. 1989. Genetic structure and divergence of hypogean and epigean Cyprinid fish. 2nd Congr. European Soc. Evolut. Biology, Rome 1989, Abstracts: 18.

COHAN, F. M. 1984. Can uniform selection retard random genetic divergence between isolated conspecific populations? Evolution 38: 495-504.

CROUAU-ROY, B. 1987. Spéciation et structure génétique des populations chez les coléopteres Speonomus. Mém. Biospéol. 14 (41): 1-312.

CULVER, D. C. 1982. Cave life. Harvard University Press, Cambridge, Massachusetts.

DELAY, B., V. SBORDONI, M. COBOLLI SBORDONI, and E. DE MATTHAEIS, 1980. Divergences génétiques entre les populations de Speonomus delarouzeei du Massif du Canigou (Coleoptera Bathysciinae). Mém. Biospéol. 7: 235-247.

DE MATTHAEIS, E., R. COLOGNOLA, V. SBORDONI, M. COBOLLI SBORDONI, and G. L. PESCE. 1982. Genetic differentiation and variability in cave dwelling and brackish water populations of Mysidacea (Crustacea). Z. f. Zool. Systematik u. Evolutionsforschung 20: 198-208.

HARRIS, H., and D. A. HOPKINSON. 1978. Handbook of enzyme electrophoresis in human genetics. Supplement. North-Holland Publ. Co., Amsterdam.

HIGHTON, R., and T. P. WEBSTER. 1976. Geographic protein variation and divergence in populations of the salamander Plethodon cinereus. Evolution 30: 33-45.

HOLTHUIS, L. B. 1986. Decapoda. p. 489. In L. Botosaneanu (ed.), Stygofauna Mundi. Brill E. J., Leiden.

HOWARTH, F. G. 1987. The evolution of non-relictual tropical troglobites. Int. J. Speleol. 16: $1-16$.

JUBERTHIE, C. 1988. Paleoenvironment and speciation in the cave beetle complex Speonomus delarouzeei (Coleoptera, Bathysciinae). Int. J. Speleol. 17: 31-50.

JUBERTHIE-JUPEAU, L. 1988. Mating behaviour and barriers to hybridization in the cave beetle of the Speonomus delarouzeei complex (Coleoptera, Catopidae, Bathysciinae). Int. J. Speleol. 17: 51-63.

KNOWLTON, N. 1986. Cryptic and sibling species among the Decapod Crustacea. Journal of Crustacean Biology 6 (3): 356-363.

LAING, C. D., G. R. CARMODY, and S. B. PECK. 1976. How common are sibling species in cave-inhabiting invertebrates? Am. Nat. 110: 184-189.

NEI, M. 1975. Molecular population genetics and evolution. North Holland, Amsterdam.

NEI, M. 1978. Estimation of average heterozygosity and genetic distance from a small number of individuals. Genetics, 89: 583-590.

NELSON, K., and D. HEDGECOCK. 1980. Enzyme polymorphism and adaptative stategy in the decapod crustacea. Am. Nat. 116:238-280.

NEVO, E., A. BEILES, and R. BEN-SHLOMO. 1984. The evolutionary significance of genetic diversity: ecological, demographic and life history correlates. In G.S. Mani (ed.), Dynamics of genetic diversity. Lecture Notes in Biomathematic 53: 13-213. 
POULIK, M. D. 1957. Starch electrophoresis in a discontinuous system of buffer. Nature 180: 1447-1479.

PRAKASH, S., R. C. LEWONTIN, and J. L. HUBBY. 1969. A molecular approach to the study of genic heterozygosity in natural populations. IV. Patterns of genic variation in central, marginal and isolated populations of Drosophila pseudobscura. Genetics 61: 841-858.

ROUCH, R. and D. L. DANIELOPOL. 1987. L'origine de la faune souterraine, entre le paradigme du refuge et le modèle de la colonisation active. Stygologia 3 (4): 345-472.

SBORDONI, V. 1982. Advances in Speciation of Cave Animals. pp. 219-240. In C. Barigozzi (ed.), Mechanisms of Speciation. Alan R. Liss, New York.

SBORDONI, V., M. COBOLLI SBORDONI, and E. DE MATTHAEIS. 1979. Divergenza genetica tra popolazioni e specie ipogee ed epigee di Niphargus (Crustacea, Amphipoda). Lavori Soc. Ital. Biogeografia (N.S.) 6 (Perugia 1976): 1-23.

SBORDONI, V., A. CACCONE, E. DE MATTHAEIS, and M. COBOLLI SBORDONI. 1980. Biochemical divergence between cavernicolous and marine Sphaeromidae and the Mediterraenan salinity crisis. Experientia 36: 48-49.

SBORDONI, V., G. ALLEGRUCCI, D. CESARONI, M. COBOLLI SBORDONI, and E. DE MATTHAEIS. 1985. Genetic structure of populations and species of Dolichopoda cave crickets: evidence of peripatric divergence. Boll. Zool. 52: 139-156.

SBORDONI, V., G. ALLEGRUCCI, A. CACCONE, G. CARCHINI, and D. CESARONI. 1987 Microevolutionary studies in Dolichopodinae cave crickets. pp. 514-540. In Baccetti B.M. (ed.), Evolutionary Biology of Orthopteroid Insects, Ellis Horwood Limited, Chichester.

SBORDONI, V., A. CACCONE, G. ALLEGRUCCI, and D. CESARONI. 1990. Molecular island Biogeography. Atti Convegn. Lincei, 85: 55-83.

SELANDER, R. K., M. H. SMITH, S. Y. YANG, W. E. JONSON, and J. B. GENTRY. 1971. Biochemical polymorphism and systematics in the genus Peromyscus. I. Variation in the old-field mouse Peromyscus polionotus. Studies in Genetics, VI Univ. Texas Publ. 7103: 49-90.

SHANNON, C. E., and W. WEAVER. 1963. The mathematical theory of communication. Univ. Illinois Press, Urbana.

SHAW, C. R., and R. PRASAD. 1970. Starch gel electrophoresis of enzymes. A compilation of recipes. Biochem. Genet. 4: 297-320.

SKET, B. 1971. Uber Struktur und Herkunft der unterdishen Fauna Jugoslawiens. Biol. vestnik 18: 69-78.

SKET, B. 1985. Why all cave animals do not look alike- A discussion on adaptive value of reduction processes. NSS Bulletin. 47 (2): 78-85.

SNEATH, P. H. A., and R. R. SOKAL. 1973. Numerical Taxonomy. Freeman, San Francisco.

SPENCER, N., D. A. HOPKINSON, and H. HARRIS. 1968. Adenosine deaminase polymorphism in man. Annals of Human Genetics 32: 9-14.

STOCK, J. 1967. The taxonomy and zoogeography of the Hadziid Amphipoda with emphasis on the west indian taxa. Studies on the fauna of Curacao and the other Caribbean Islands, 177.

WARD, R. D., and J. A. BEARDMORE. 1977. Protein variation in the plaice, Pleuronectes platessa. Genet. Res. 30: 45-62. 